\title{
Correction to: Actigraphy assessments of circadian sleep-wake cycles in the Vegetative and Minimally Conscious States
}

D. Cruse*, A. Thibaut, A. Demertzi, J. C. Nantes, M. A. Bruno, O. Gosseries, A. Vanhaudenhuyse, T. A. Bekinschtein, A. M. Owen and S. Laureys

\section{Correction}

The original article [1] contains an error affecting the actigraphy time-stamps throughout the article, particularly in Table 1.

All data for this manuscript were collected in Liège, Belgium, and exported and analysed in London, Ontario. The authors have recently discovered that a software error occurred during data export that caused all actigraphy time-stamps to be shifted by exactly 6-hours due to the time-zone difference between Belgium and Ontario. As a result, the acrophase values reported in the original Table 1 should all be read as occurring 6-hours earlier in the day as displayed in the corrected Table 1 ahead (for example, if an acrophase is reported as 16:57 in Table 1, it should be read as 10:57). The corrected Table 1 appears as within this Erratum.

Importantly, as this software error added only a constant value to each time-stamp of each patient data set, no analyses or results are affected.

Received: 23 July 2018 Accepted: 25 July 2018

Published online: 11 August 2018

\section{Reference}

1. Cruse D, et al. Actigraphy assessments of circadian sleep-wake cycles in the Vegetative and Minimally Conscious States. BMC Med. 2013;11:18. https://doi.org/10.1186/1741-7015-11-18

\footnotetext{
* Correspondence: d.cruse@bham.ac.uk

Brain and Mind Institute, University of Western Ontario, 1151 Richmond

Street, London, ON N6A 3K7, Canada
} 
Table 1 Demographics and circadian rhythm fits for all patients

\begin{tabular}{|c|c|c|c|c|c|c|c|c|c|c|}
\hline Patient ID & Gender & Age (Years) & $\begin{array}{l}\text { Post-Ictus } \\
\text { (Months) }\end{array}$ & Diagnosis & Etiology & CRS-R & Mesor & Acrophase & Amplitude & Sig. Fit? \\
\hline 1 & M & 53 & 40 & MCS & Non-TBI & 11 & 9.56 & $10: 57$ & 6.7 & Yes \\
\hline 2 & M & 31 & 22 & MCS & Non-TBI & 13 & 8.67 & $15: 44$ & 10.46 & Yes \\
\hline 3 & W & 30 & 78 & MCS & Non-TBI & 9 & 15.84 & $9: 45$ & 13.58 & Yes \\
\hline 4 & M & 31 & 16 & MCS & Non-TB| & 7 & 5.51 & $13: 27$ & 4.63 & Yes \\
\hline 5 & M & 27 & 50 & MCS & Non-TBI & 9 & 31.01 & $9: 43$ & 9.94 & No \\
\hline 6 & W & 36 & 17 & MCS & Non-TB| & 13 & 21.25 & 13:10 & 15.87 & Yes \\
\hline 7 & M & 34 & 35 & MCS & Non-TBI & 12 & 26.4 & 11:35 & 28.34 & Yes \\
\hline 8 & W & 63 & 3 & MCS & Non-TBI & 13 & 6.32 & $10: 36$ & 6.77 & Yes \\
\hline 9 & M & 57 & 12 & MCS & Non-TB| & 7 & 12.89 & $11: 26$ & 9.4 & Yes \\
\hline 10 & M & 66 & 2 & MCS & Non-TB| & 10 & 34.18 & $16: 25$ & 26 & Yes \\
\hline 11 & M & 11 & 48 & MCS & Non-TBI & 13 & 32.4 & 13:05 & 35.79 & Yes \\
\hline 12 & W & 43 & 3 & MCS & Non-TBI & 6 & 9.12 & 11:49 & 8.76 & Yes \\
\hline 13 & W & 34 & 256 & MCS & Non-TBI & 12 & 20.63 & $12: 40$ & 15.36 & Yes \\
\hline 14 & M & 30 & 106 & MCS & $\mathrm{TBI}$ & 14 & 49.2 & 14:18 & 41.91 & Yes \\
\hline 15 & W & 21 & 1 & MCS & $\mathrm{TB} \mid$ & 10 & 17.23 & $11: 57$ & 12.52 & Yes \\
\hline 16 & M & 46 & 17 & MCS & $\mathrm{TB} \mid$ & 11 & 42.8 & $16: 46$ & 48.47 & Yes \\
\hline 17 & M & 30 & 27 & MCS & $\mathrm{TBI}$ & 10 & 20.71 & $14: 12$ & 17.98 & Yes \\
\hline 18 & M & 30 & 13 & MCS & $\mathrm{TB} \mid$ & 9 & 54.24 & 11:16 & 22.6 & Yes \\
\hline 19 & M & 24 & 10 & MCS & $\mathrm{TBI}$ & 10 & 114.11 & $12: 50$ & 65.35 & Yes \\
\hline 20 & W & 75 & 9 & MCS & $\mathrm{TBI}$ & 9 & 8.57 & 11:06 & 9.55 & Yes \\
\hline 21 & W & 34 & 99 & MCS & $\mathrm{TBI}$ & 12 & 18.22 & $9: 46$ & 6.64 & No \\
\hline 22 & W & 27 & 41 & MCS & $\mathrm{TBI}$ & 11 & 15.02 & $17: 05$ & 16.01 & Yes \\
\hline 23 & M & 24 & 88 & MCS & $\mathrm{TB} \mid$ & 11 & 18.54 & 13:49 & 14.62 & No \\
\hline 24 & M & 44 & 287 & MCS & $\mathrm{TB} \mid$ & 9 & 5.25 & 13:37 & 4.83 & Yes \\
\hline 25 & W & 30 & 4 & MCS & $\mathrm{TBI}$ & 9 & 10.41 & $11: 29$ & 13.41 & Yes \\
\hline 26 & M & 34 & 33 & MCS & $\mathrm{TBI}$ & 8 & 27.22 & $12: 30$ & 27.89 & No \\
\hline 27 & M & 23 & 10 & MCS & $\mathrm{TBI}$ & 10 & 8.47 & $10: 38$ & 7.74 & Yes \\
\hline 28 & M & 27 & 37 & MCS & $\mathrm{TBI}$ & 13 & 40.74 & $14: 53$ & 34.18 & Yes \\
\hline 29 & M & 61 & 4 & MCS & $\mathrm{TBI}$ & 10 & 21.8 & $15: 41$ & 19.72 & Yes \\
\hline 30 & M & 24 & 24 & MCS & $\mathrm{TBI}$ & 11 & 88.98 & $16: 18$ & 56.45 & No \\
\hline 31 & M & 23 & 66 & MCS & $\mathrm{TBI}$ & 16 & 14.09 & $11: 24$ & 18.96 & No \\
\hline 32 & $M$ & 21 & 38 & MCS & $\mathrm{TBI}$ & 8 & 10.09 & $9: 55$ & 7.06 & Yes \\
\hline 33 & M & 30 & 109 & MCS & $\mathrm{TBI}$ & 10 & 35.15 & $14: 26$ & 21.87 & Yes \\
\hline 34 & W & 24 & 21 & MCS & $\mathrm{TBI}$ & 10 & 15.45 & $12: 58$ & 18.08 & Yes \\
\hline 35 & M & 36 & 4 & MCS & $\mathrm{TB} \mid$ & 11 & 6.49 & 10:10 & 5.53 & Yes \\
\hline 36 & M & 65 & 22 & MCS & $\mathrm{TBI}$ & 7 & 11.3 & $10: 13$ & 15.14 & Yes \\
\hline 37 & M & 21 & 5 & MCS & $\mathrm{TBI}$ & 7 & 9.69 & $7: 41$ & 7.9 & Yes \\
\hline 38 & W & 66 & 0 & VS & Non-TBI & 3 & 4.56 & $9: 43$ & 5.24 & Yes \\
\hline 39 & M & 35 & 220 & VS & Non-TBI & 7 & 11.01 & $13: 20$ & 10.24 & Yes \\
\hline 40 & M & 30 & 24 & VS & Non-TBI & 6 & 25.15 & $9: 28$ & 14.67 & Yes \\
\hline 41 & W & 48 & 15 & VS & Non-TBI & 5 & 8.5 & 11:14 & 10.53 & Yes \\
\hline 42 & W & 67 & 45 & VS & Non-TBI & 5 & 15.46 & $12: 29$ & 12.23 & Yes \\
\hline 43 & M & 53 & 1 & VS & Non-TBI & 5 & 9.53 & $10: 53$ & 7.92 & Yes \\
\hline
\end{tabular}


Table 1 Demographics and circadian rhythm fits for all patients (Continued)

\begin{tabular}{|c|c|c|c|c|c|c|c|c|c|c|}
\hline Patient ID & Gender & Age (Years) & $\begin{array}{l}\text { Post-lctus } \\
\text { (Months) }\end{array}$ & Diagnosis & Etiology & CRS-R & Mesor & Acrophase & Amplitude & Sig. Fit? \\
\hline 44 & $M$ & 34 & 17 & VS & Non-TBI & 7 & 5.68 & $11: 43$ & 2.79 & Yes \\
\hline 45 & W & 41 & 56 & VS & Non-TBI & 5 & 14.39 & $11: 35$ & 14.49 & Yes \\
\hline 46 & W & 48 & 4 & VS & Non-TB| & 4 & 4.78 & $14: 02$ & 3.44 & No \\
\hline 47 & M & 48 & 30 & VS & Non-TBI & 6 & 2.31 & 11:19 & 2.03 & Yes \\
\hline 48 & M & 36 & 66 & VS & Non-TBI & 5 & 7.34 & $11: 40$ & 6.88 & Yes \\
\hline 49 & M & 34 & 43 & VS & $\mathrm{TB} \mid$ & 6 & 10.84 & $7: 47$ & 7.87 & Yes \\
\hline 50 & W & 30 & 18 & VS & $\mathrm{TB} \mid$ & 4 & 6.49 & $10: 56$ & 8.07 & Yes \\
\hline 51 & M & 21 & 7 & VS & $\mathrm{TB} \mid$ & 7 & 8.58 & $12: 15$ & 8.29 & Yes \\
\hline 52 & M & 35 & 290 & VS & TBI & 8 & 57.65 & $17: 10$ & 36.56 & No \\
\hline 53 & M & 21 & 8 & VS & $\mathrm{TBI}$ & 6 & 9.95 & $13: 22$ & 6.45 & Yes \\
\hline 54 & M & 13 & 1 & VS & $\mathrm{TB} \mid$ & 6 & 5.78 & $15: 24$ & 3.12 & No \\
\hline 55 & M & 25 & 15 & VS & TBI & 5 & 10.02 & 10:19 & 9.05 & Yes \\
\hline
\end{tabular}

The final column indicates whether the circadian rhythm fit was significant or not. MCS Minimally Conscious State, TBI Traumatic Brain Injury, VS Vegetative State 